\title{
Genotype-dependent gene expression profile of the antioxidant defense system (ADS) in the liver of a GH-transgenic zebrafish model
}

\author{
Carlos E. da Rosa • Márcio A. Figueiredo • \\ Carlos F. C. Lanes · Daniela V. Almeida • \\ Luis F. Marins
}

Received: 2 November 2009/Accepted: 1 April 2010/Published online: 24 April 2010

(C) Springer Science+Business Media B.V. 2010

\begin{abstract}
The aim of this study was to evaluate the effects of growth hormone $(\mathrm{GH})$ overexpression on the gene expression profile of multiple components of the antioxidant defense system (ADS) of different genotypes of a GH-transgenic zebrafish (Danio rerio) model. Several ADS-related genes were analyzed by semiquantitative reverse transcription-PCR in the liver of hemizygous (HE) and homozygous (HO) transgenic zebrafish. The results showed a significant reduction in the glutamate cysteine ligase catalytic subunit (GCLC) and the gene expression of two glutathione $S$-transferase (GST) isoforms and an increase in the glutathione reductase gene in the $\mathrm{HO}$ group compared to non-transgenic controls. The
\end{abstract}

C. E. da Rosa · L. F. Marins ( $₫)$

Instituto de Ciências Biológicas, Universidade Federal do Rio Grande (FURG), Avenida Itália, Km 8, Rio Grande, RS 96201-900, Brazil

e-mail: dqmluf@furg.br

D. V. Almeida · L. F. Marins

Programa de Pós-Graduação em Ciências FisiológicasFisiologia Animal Comparada, Instituto de Ciências

Biológicas, FURG, Rio Grande, RS 96201-900, Brazil

M. A. Figueiredo · L. F. Marins

Programa de Pós-Graduação em Aqüicultura-Instituto de Oceanografia, FURG, Rio Grande, RS 96201-900, Brazil

C. F. C. Lanes

Faculty of Biosciences and Aquaculture, Bod $\varnothing$ University College, 8049 Bodø, Norway expression of the $\mathrm{Cu}$, $\mathrm{Zn}$-superoxide dismutase (SOD1) and catalase (CAT) genes was reduced in $\mathrm{HO}$ and HE groups, respectively. Among the ten genes analyzed, two were altered in HE transgenic zebrafish and five were altered in $\mathrm{HO}$ transgenic zebrafish. These findings indicate a genotypedependent gene expression profile of the ADS-related genes in the liver of our GH-transgenic zebrafish model and are in agreement with the general effects of GH hypersecretion in the fish and mouse, which involves a reduction in the capability of the tissues to deal with oxidative stress situations. The GH-transgenic zebrafish model used here seems to be an interesting tool for analyzing the effect of different GH expression levels on physiological processes.

Keywords Antioxidant defense system . Gene expression - Growth hormone . RT-PCR · Transgenic zebrafish

\section{Introduction}

It is well established that an overexpression of growth hormone $(\mathrm{GH})$ causes an enhancement of the metabolic rate in GH-transgenic Atlantic salmon (Deitch et al. 2006) and GH-transgenic Nile tilapia (McKenzie et al. 2003). The oxidative metabolism is a continuous source of reactive oxygen species (ROS) that can 
damage most cellular components, leading to cell death. To protect cells from these highly reactive intermediates, living organisms possess an antioxidant defense system (ADS) comprising a ROS scavenging mechanism of both enzymatic and non-enzymatic antioxidants (Halliwell and Gutteridge 1999). Small variations in the basal level of intracellular ROS have been shown to modulate cell metabolism, gene expression, and the post-translational modification of proteins (Dalton et al. 1999).

Even though $\mathrm{GH}$ accelerates metabolism and increases ROS production, its administration or overexpression surprisingly causes a reduction in the activity of multiple ADS compounds, including glutathione metabolism in mammalian models (BrownBorg et al. 2005; Brown-Borg 2009). GH appears to be involved in the regulation of these pathways by changing enzyme expression, substrate availability, and the redox potential of the tissues and whole organism (Brown-Borg et al. 2005). Our group has recently developed a stable transgenic zebrafish (Danio rerio) model (named the F0104 lineage) which overexpresses the marine silverside (Odonthestes argentinensis) GH cDNA under strong transcriptional control of the carp (Cyprinus carpio) $\beta$-actin promoter (Figueiredo et al. 2007). Using this model, Rosa et al. (2008) observed an induction in the metabolic rate and a consequent increase in ROS production in the muscle of homozygous (HO) transgenic zebrafish as well as reduced expression of the glutamate cysteine ligase catalytic subunit (GCLC) gene. Considering that the liver is an important site of ADS activity, we have evaluated the effects of $\mathrm{GH}$ overexpression in this particular tissue, analyzing a wider range of ADSrelated genes in different genotypes of GH-transgenic zebrafish from the F0104 lineage.

\section{Materials and methods}

Four-month-old non-transgenic (NT), hemizygous (HE), and HO transgenic fish were obtained and reared as described by Rosa et al. (2008). The mortality rate for all three groups was recorded during the maintenance period.

The liver from five animals per group were dissected and total RNA extracted and used for semiquantitative reverse transcription (RT)-PCR, essentially as described by Rosa et al. (2008). The glyceraldehyde 6-phosphate (GAPDH) and $\beta$-actin genes were used as internal controls for data normalization. Since both internal controls have the same expression pattern (data not shown), the results presented here are relative to those obtained for GAPDH normalization only. The number of cycles for each gene was previously determined in order to avoid the PCR plateau phase. Gene-specific primers, GenBank accession number, and number of PCR cycles used for each gene are given in Table 1. The ADS-related genes analyzed were the GCLC, glutathione reductase (GR), glutathione peroxidase (GPx1a), glutathione $S$ - transferase $\alpha 1$ (GST- $\alpha 1$ ), glutathione $S$ - transferase $\mu$ (GST- $\mu$ ), glutathione $S$ - transferase $\pi$ (GST- $\pi$ ), glutathione $S$-transferase $\rho$ (GST- $\rho$ ), Cu,Zn-superoxide dismutase (Cu,Zn-SOD or SOD1), Mn-superoxide dismutase (Mn-SOD or SOD2), and catalase (CAT). All PCR reactions were carried out in a $12.5-\mu$ l reaction volume containing $1.25 \mu \mathrm{l}$ of $10 \times$ PCR buffer (Invitrogen, Brazil), $0.25 \mu \mathrm{M}$ of each primer, $0.25 \mathrm{mM}$ of each dNTP, 0.5 U Platinum Taq DNA polymerase (Invitrogen), and $0.5 \mu \mathrm{l}$ of cDNA solution. The reaction was incubated at $94^{\circ} \mathrm{C}$ for $2 \mathrm{~min}$, followed by a predetermined number of cycles of $30 \mathrm{~s}$ at $94^{\circ} \mathrm{C}, 30 \mathrm{~s}$ at $60^{\circ} \mathrm{C}$, and $1 \mathrm{~min}$ and $30 \mathrm{~s}$ at $72^{\circ} \mathrm{C}$, with a final step of $10 \mathrm{~min}$ at $72^{\circ} \mathrm{C}$. PCR products were analyzed on $1 \%$ agarose gels, photographed, and the bands quantified by densitometry using the software 1DScan EX 3.1 (Scanalitics, Fairfax, VA).

The gene expression data were analyzed as fold induction, with the expression of each gene in the NT group being given a value of 1 . Data were submitted to one-way variance analysis (ANOVA) followed by a post hoc means comparison test (Tukey's test). Analysis assumptions (normality and variance homogeneity) were previously verified. The adopted alpha was 0.05 . Results are expressed as the mean \pm standard error (SE).

\section{Results}

The mortality rates of $\mathrm{HO}$ zebrafish were significantly higher $(10.18 \% \pm 1.66)$ than those observed for HE $(3.0 \% \pm 1.52)$ and NT zebrafish $(2.42 \% \pm 1.47)$. In terms of glutathione metabolism, the gene expression of GCLC in HO and HE zebrafish was significantly reduced relative to that in NT fish (Fig. 1). The 
Table 1 Gene-specific primers used for reverse transcription-PCR analysis, GenBank accession numbers of the sequences used in primer design, and number of cycles in PCR reactions

\begin{tabular}{lllll}
\hline Gene & GenBank accession no. & PCR cycles & Primers & \\
\cline { 4 - 5 } & & & Forward $\left(5^{\prime}-3^{\prime}\right)$ & Reverse $\left(5^{\prime}-3^{\prime}\right)$ \\
\hline GCLC & BC068331 & 25 & aggcctgagtatggcagcta & gtggtccgattcgttctcat \\
GR & XM7043891 & 32 & tgaaaagggcaaaattgagttta & tttcgagaggtaatggcgtaata \\
GPx1a & BC083461 & 32 & gaagaaatcctgcagtctctgaa & gaaccttctgctgtacctcttga \\
GST- $\alpha 1$ & BC060914 & 25 & cgcaggaaaatacaacctctatg & agcttccagaagatgaacatcag \\
GST- $\mu$ & BC057526 & 25 & gaatacaccggtaccaaatacga & ctcaaagtgatccaggaaacatc \\
GST- $\pi$ & BI979167 & 25 & cagttgcctaaatttgaagatgg & agcttccagaagatgaacatcag \\
GST- $\rho$ & BC139572 & 28 & tgcctttatctggagagtgtgtt & tactccatcagtctgggacatct \\
SOD1 & BC055516 & 25 & caccgtctattcaatcaagagg & agaatgttggcctgacaaagtta \\
SOD2 & BC060895 & 23 & tctcctgacctcacatatgact & tggcagctgatatcttctctttc \\
CAT & BC051626 & 29 & aacaacaccccatcttcttat & atgtgtgtctgggtaggagaaaa \\
GAPDH & BC095386 & 25 & gatggtcatgcaatcacagtcta & atcatacttggcaggttctcaa \\
\hline
\end{tabular}

GCLC, Glutamate cysteine ligase catalytic subunit; GR, glutathione reductase; GPx1a, glutathione peroxidase; GST- $\alpha 1$, glutathione $S$ - transferase $\alpha 1$; GST- $\mu$, glutathione $S$ - transferase $\mu$; GST- $\pi$, glutathione $S$ - transferase $\pi$; GST- $\rho$, glutathione $S$-transferase $\rho$; SOD1, Cu,Zn-superoxide dismutase; SOD2, Mn-superoxide dismutase; CAT, catalase; GAPDH, glyceraldehyde 6-phosphate

induction of GR expression in HO zebrafish was also significantly reduced relative to GR expression in NT fish (Fig. 1). The expression of the GPx gene in transgenic zebrafish was not altered when compared to that in NT fish.

Evaluation of GST and its isoforms revealed that there was a significant reduction in the gene expression of GST- $\alpha 1$ and GST- $\mu$ in HO zebrafish relative to NT and HE fish (Fig. 2). The gene expression of

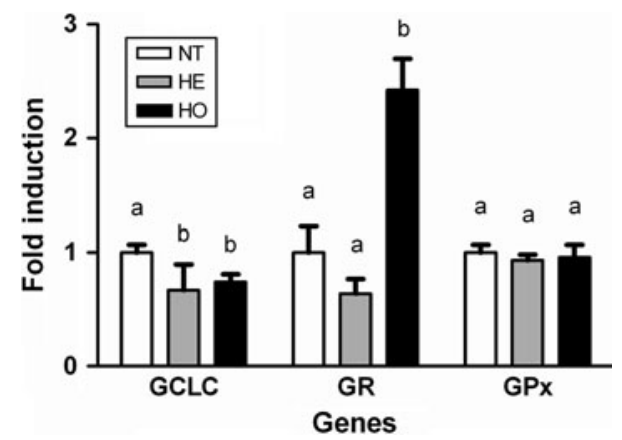

Fig. 1 Liver expression profiles of glutamate cysteine ligase catalytic subunit (GCLC), glutathione reductase (GR), and glutathione peroxidase (GPx) genes from 4-month-old nontransgenic (NT), hemizygous (HE), and homozygous (HO) growth hormone $(\mathrm{GH})$-transgenic zebrafish (Danio rerio). Data are presented as the mean \pm standard error (SE). Different letters represent significant differences $(P<0.05)$ both the $\pi$ and $\rho$ isoforms of GST was not altered in transgenic zebrafish compared to NT fish. Expression of the SOD1 gene was negatively regulated in HO zebrafish compared with that in the NT and HE fish, while the expression of SOD2 was not altered (Fig. 3). A significant reduction in the expression of the CAT gene was observed in HE zebrafish relative to NT and HO fish (Fig. 3).

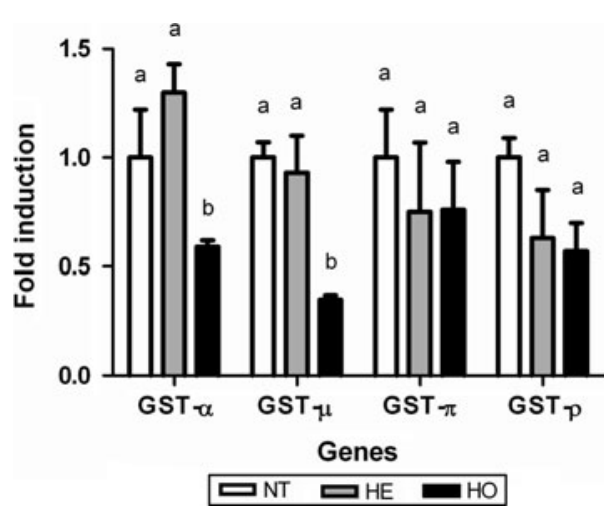

Fig. 2 Liver expression profiles of glutathione $S$-transferase isoforms [glutathione $S$-transferase $\alpha 1$ (GST- $\alpha 1)$, glutathione $S$ - transferase $\mu$ (GST- $\mu$ ), glutathione $S$ - transferase $\pi$ (GST- $\pi$ ), glutathione $S$-transferase $\rho$ (GST- $\rho)$ ] from 4-month-old NT, $\mathrm{HE}$, and HO GH-transgenic zebrafish (D. rerio). Data are presented as the mean $\pm \mathrm{SE}$. Different letters represent significant differences $(P<0.05)$ 


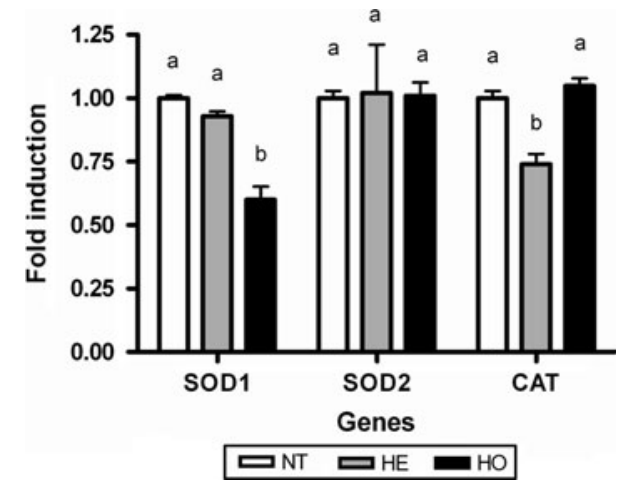

Fig. 3 Liver expression profiles of $\mathrm{Cu}, \mathrm{Zn}$-superoxide dismutase (SOD1), Mn-superoxide dismutase (SOD2), and catalase (CAT) genes from 4-month-old NT, HE, and HO GH-transgenic zebrafish (Danio rerio). Data are presented as the means \pm SE. Different letters represent significant differences $(P<0.05)$

\section{Discussion}

Overstimulation of the somatotrophic axis leads to an enhanced metabolic activity, enhancement of ROS production, and a suppressive effect on multiple components of the ADS (Brown-Borg 2009). The tripeptide glutathione (GSH) is the most important low-molecular-weight thiol found in mammalian cells, and it is considered the cell's first line of defense against ROS (Griffith 1999). The balance between its synthesis, utilization, and recycling is important to the maintenance of the redox state of the cell (Halliwell and Gutteridge 1999). The enzyme glutamate cysteine ligase is the first and rate-limiting step in GSH formation, and this enzyme is composed of both a modulatory (GCLM) and a catalytic subunit (GCLC) (Griffith 1999). In this study, we have demonstrated that an excess of GH negatively affects the expression of the GCLC gene in the liver of both $\mathrm{HE}$ and $\mathrm{HO} \mathrm{GH}$-transgenic zebrafish. Muscle tissue from transgenic animals of this same lineage has recently been reported to have the same pattern of response (Rosa et al. 2008). In coho salmon (Oncorhynchus kisutch), GH transgenesis induced a significant reduction in GCLC hepatic activity compared with NT fish (Leggatt et al. 2007). These results indicate that the GH negatively affects the activity and gene expression of this enzyme in fishes.

The enzyme GR acts by recycling the oxidized glutathione (GSSG) to two GSH molecules at the expense of NADPH (Halliwell and Gutteridge 1999).
We observed that GR gene transcription was significantly augmented in $\mathrm{HO}$ zebrafish compared to the other two groups (NT and HE). Increased GR activity was also observed in the liver of GH-transgenic coho salmon (Leggatt et al. 2007); the authors related this increase to the GSH recycling process.

In terms of the GSH spending process, the GST enzymes act to conjugate GSH to a broad range of endogenous and exogenous compounds (Halliwell and Gutteridge 1999). Its isoforms are classified by their differences in amino acid sequences, substrate specificities, and immunological properties (Srivastava and Waxman 1993). Rats injected with GH were found to have a reduced expression of both the $\alpha$ and $\mu$ isoforms of GST (Srivastava and Waxman 1993; Staffas et al. 1998). We also observed a reduction in the gene expression of the $\alpha$ and $\mu$ GST isoforms in the $\mathrm{HO}$ transgenic zebrafish. These results are in agreement with those observed for the liver, kidney, and brain of the Ames dwarf mice treated with $\mathrm{GH}$ (Brown-Borg et al. 2005), indicating a reduction in the capability of these animals to conjugate GSH in order to maintain the cellular redox state.

It is expected that under condition of high ROS production, an organism will counteract the deleterious effects of these ROS by inducing antioxidant enzymes. SOD degrades the superoxide anion $\left(\mathrm{O}_{2}{ }^{-}\right)$, generating $\mathrm{H}_{2} \mathrm{O}_{2}$, which in turn is degraded by CAT. An imbalance in the activities of this pair of enzymes would increase oxidative stress in the organism (Halliwell and Gutteridge 1999). Tissues from mice with high plasma GH concentration have been found to exhibit significantly reduced levels of antioxidant enzymes, including SOD1, SOD2, CAT, and GPx (Hauck and Bartke 2000; Brown-Borg and Rakoczy 2003). More directly, in vitro effects of GH and IGF1 (insulin-like growth factor 1) strongly support the in vivo data, showing that these two hormones downregulate CAT, GPx, and SOD2 in mouse hepatocytes (Brown-Borg 2009). Although the HO transgenic zebrafish from the F0104 lineage possesses the ability for higher ROS production, its muscle SOD and CAT activity are unaltered (Rosa et al. 2008). In this study, we observed that liver SOD1 gene expression was in fact downregulated in the $\mathrm{HO}$ group and that the CAT gene expression was diminished in $\mathrm{HE}$ animals, indicating a genotype-dependent response.

In conclusion, the results reported here demonstrate a genotype-dependent expression profile of 
ADS-related genes in the liver of a GH-transgenic zebrafish model. Among the ten ADS-related genes analyzed, two (GCLC and CAT) were altered in HE transgenic zebrafish, while five (GCLC, GR, GST- $\alpha$ 1, GST- $\mu$, and SOD1) were altered in HO transgenic zebrafish. These findings may be related to the high mortality rate observed in $\mathrm{HO}$ group. It is important to emphasize that the overall oxygen consumption is strongly affected only in the HO group (Rosa et al. 2008) and that differences in the expression of ADSrelated genes could be a consequence of this effect. Finally, the GH-transgenic zebrafish model used here would appear to be an interesting tool for analyzing the effect of different levels of $\mathrm{GH}$ expression on physiological processes since homozygous animals have a twofold higher quantity of $\mathrm{GH}$ than their hemizygous siblings.

Acknowledgments This work was supported by the Brazilian agencies $\mathrm{CNPq}$ (Conselho Nacional de Desenvolvimento Científico e Tecnológico) and CAPES (Coordenação de Aperfeiçoamento de Pessoal de Nível Superior). The experiments comply with current Brazilian laws and regulations.

\section{References}

Brown-Borg HM (2009) Hormonal control of aging in rodents: the somatotrophic axis. Exp Gerontol 44:10-19

Brown-Borg HM, Rakoczy SG (2003) Growth hormone administration to long-living dwarf mice alters multiple components of the antioxidative defense system. Mech Ageing Dev 124:1013-1124

Brown-Borg HM, Rakoczy SG, Uthus EO (2005) Growth hormone alters methionine and glutathione metabolism in Ames dwarf mice. Mech Ageing Dev 126:389-398
Dalton TP, Shertzer HG, Puga A (1999) Regulation of gene expression by reactive oxygen. Annu Rev Pharmacol Toxicol 39:67-101

Deitch EJ, Fletcher GL, Petersen LH, Costa IASF, Shears MA, Driedzic WR, Gamperl AK (2006) Cardiorespiratory modifications, and limitations, in post-smolt growth hormone transgenic Atlantic salmon Salmo salar. J Exp Biol 209:1310-1325

Figueiredo MA, Lanes CFC, Almeida DV, Proietti MC, Marins LF (2007) Improving the production of transgenic fish germline: in vivo mosaicism evaluation by GFP transgene co-injection strategy. Genet Mol Biol 30:31-36

Griffith OW (1999) Biologic and pharmacologic regulation of mammalian glutathione synthesis. Free Radic Biol Med 27:922-935

Halliwell B, Gutteridge JM (1999) Free radicals in biology and medicine. Oxford University Press, New York

Hauck SJ, Bartke A (2000) Effects of growth hormone on hypothalamic catalase and $\mathrm{Cu} / \mathrm{Zn}$ superoxide dismutase. Free Rad Biol Med 28:970-978

Leggatt RA, Brauner CJ, Iwama GK, Devlin RH (2007) The glutathione antioxidant system is enhanced in growth hormone transgenic coho salmon (Oncorhynchus kisutch). J Comp Physiol B 177:413-422

McKenzie DJ, Martínez R, Morales A, Acosta J, Morales R, Taylor EW, Steffensen JF, Estrada MP (2003) Effects of growth hormone transgenesis on metabolic rate, exercise performance and hypoxia tolerance in tilapia hybrids. J Fish Biol 63:398-409

Rosa CE, Figueiredo MA, Lanes CFC, Almeida DV, Monserrat JM, Marins LF (2008) Metabolic rate and reactive oxygen species production in different genotypes of GH-transgenic zebrafish. Comp Biochem Physiol B 149:209-214

Srivastava PK, Waxman DJ (1993) Sex-dependent expression and growth hormone regulation of class alpha and class mu glutathione S-transferase mRNAs in adult rat liver. Biochem J 294:159-165

Staffas L, Ellis EM, Hayes JD, Lundgren B, Depierre JW, Mankowitz L (1998) Growth hormone- and testosteronedependent regulation of glutathione transferase subunit A5 in rat liver. Biochem J 332:763-768 